\title{
Moral de la transgresión, vigencia de un antiguo orden ${ }^{1}$
}

\author{
ALICIA H. PULEO \\ Universidad de Valladolid
}

RESUMEN. En los límites de este estudio es imposible hacer referencia a todos los aspectos presentes en obras tales como L'Histoire de l'Érotisme o La Souveraineté. Intentaré, por lo tanto, solamente responder a las siguientes preguntas: ¿la moral de la transgresión batailleana que reconoce en Nietzsche y Sade a sus geniales inspiradores responde a una transformación del patriarcado que se hallaría actualmente en curso? ¿Su exaltación de la violencia y el crimen provendrían de un sesgo de género del pensamiento? ¿La cultura de masas y la (a menudo pseudo) cultura de élites de hoy en día son la correa de transmisión por la que se generaliza esta moral de la transgresión? ¿Qué relación existe entre la violencia irracional explícita y el crimen sexual omnipresentes en la ficción, por un lado, y una jurisprudencia moderna que busca defender de modo creciente el derecho a la seguridad y a la libertad sexual de las mujeres, por otro? En otras palabras: ¿la voluntad de aniquilación transgresiva esconde el deseo de mantenimiento de jerarquías arcaicas de género?

En una época de pérdida de los Grandes Relatos, la transgresión tiende a tomar el lugar de la denostada revolución. El acto gratuito y la rebeldía sin objetivo preciso sustituyen a la acción contestataria fundamentada en un proyecto. Bataille, como teórico de la transgresión, tiene, por lo tanto, una vigencia que va más allá de la
ABSTRACT. It is not posible to refer to all aspects of such works as L'Histoire de l'Érotisme or La Souveraineté within the bounds of this paper. Hence I shall try only the answer these questions: Does Bataille's transgression morals, which regards Nietzsche and Sade as its genial forerunners, pertain to a going-on transformation of patriarchy? Does his extolment of violence and crime arise from a thought gendre feature? Are mass culture and (often pseudo) elite culture driving belts through which such transgression morals get generalized nowadays? What is the relationship between explicit irrational violence and crime, which are present all through fiction works, and modern jurisprudence which aims at increasingly protect women's right to safety and sexual freedom? In other words, does transgressive annihilation will hide a wish to sep archaic gendre hierarchies?

cita obligada como filósofo consagrado e indiscutido (pero discutible) del erotismo.

Antes de adentrarme en el tema, recordaré la diferencia entre sexismo y androcentrismo. El sexismo es la ideología de la inferioridad de uno de los sexos. En una sociedad patriarcal $-\mathrm{y}$ todas las sociedades del pasado y del presente lo son- este 
sexo es el femenino. El androcentrismo es un punto de vista parcial masculino que hace del varón y de su experiencia la medida de todas las cosas. Por lo general, ambos elementos se encuentran juntos y confundidos en el pensamiento de los filósofos.

En un trabajo anterior ${ }^{2}$ analicé el sexismo inherente a la teoría del erotismo transgresivo de George Bataille. Ahora, a partir de algunos elementos sugeridos pero no desarrollados en aquella ocasión, me propongo dar un paso más y estudiar su carácter androcéntrico.

La moral de la transgresión propuesta por Bataille es heredera confesa del inmoralismo nietzscheano. Pero se trata de un desarrollo peculiar que se alimenta de una fuente francesa: los escritos del marqués de Sade y su particular enfoque de la sexualidad humana. En sus comentarios sobre Nietzsche (Sur Nietzsche) opone la «cumbre moral» (sommet moral) a la «decadencia» (déclin). La primera se caracteriza por el gasto descontrolado de energía y la violación de la integridad de los seres, por una exuberancia que no respeta los límites proclamados por la moral ordinaria. La decadencia, en cambio, destaca por su preocupación de conservación y enriquecimiento del ser. Es el momento de obediencia a las normas morales propio de la pérdida de las fuerzas juveniles. La vida, valor supremo, sólo puede tener lugar por el mal. Habría una contradicción entre el reconocimiento de la existencia de los seres en tanto separados como «mal» $\mathrm{y}$ la definición del «bien» como respeto del interés de los otros. La vida no es sino comunicación, fluir de energía. Vivimos sólo si consentimos entrar en el juego que pone nuestro ser y el de los demás en situación de riesgo. «Sólo si sacrifico en mí y en los demás la integridad del ser, abriéndome a la comunión, accedo a la cumbre moral. Y la cumbre no es soportar sino querer el mal. Es el acuerdo voluntario con el pecado, el crimen, el mal. Con un destino sin tregua que exige que unos mueran para que los otros vivan» ${ }^{3}$. El asceta y el burgués se encuentran, según Bataille, en las antípodas de esta moral de sacralización del instante, ya que trabajan reprimiendo sus pulsiones en aras de objetivo futuro, distinto en cada caso pero coincidentes en su carácter acumulativo, previsor. Observemos, sin embargo, que el socialismo libertario de Bataille tiene curiosas similitudes con el espíritu del capitalismo tardío propio de la sociedad del riesgo postmoderna ${ }^{4}$. El parecido se hace visible si atendemos al carácter central y la valoración positiva de las nociones de descentralización, fluidez, comunicación, asunción del azar y del riesgo, estética (por encima de la ética), consumo innecesario (dépense) y transgresión de las costumbres y normas tradicionales.

Tanto en el pensamiento de Sade como en el de Bataille, el erotismo es presentado como una vía privilegiada de acceso a la experiencia de la soberanía. L'Histoire de l'Érotisme es una respuesta a los teóricos de la revolución sexual, a esos nuevos epicúreos que pretendían abolir la vergüenza y las normas represivas que afectaban al fenómeno natural de la sexualidad humana. Al diferenciar sexualidad de erotismo, Bataille afirma que las prohibiciones morales que rodean el sexo son necesarias y no deben desaparecer so pena de extinción de la experiencia central de la soberanía en la sociedad de los iguales. «El hombre, en el acto carnal, al mancillar y mancillarse, atraviesa el límite de los seres» ${ }^{5}$. Experimenta de esa forma el absoluto, es decir, la Nada, la ausencia de sentido, la nulidad total de lo existente y el vacío del más allá.

Esta interpretación del erotismo como deseo de caída, de pérdida, como desafío de la moral judeocristiana, le valió el calificativo de puritano. La rebelión contra las normas, que no pretende abolirlas ni suplantarlas, sino transgredirlas, sería, a los ojos de Sartre, la actitud de «un nuevo 
místico» ${ }^{6}$ que no ha podido liberarse realmente de la religión de su infancia.

El enfoque de género nos abre nuevas perspectivas que, como ya he señalado, pueden orientarse hacia el análisis del sexismo y del androcentrismo de esta moral de la transgresión batailleana. En el primer caso he puesto de relieve la existencia de lo que, en palabras de Amelia Valcárcel, serían figuras de la heterodesignación ${ }^{7}$ : la prostituta, la esposa-madre, la trabajadora aparecen como formas únicas y mutuamente excluyentes de declinación de lo femenino. La trabajadora es la mujer asexuada por haber entrado en la cadena de causalidad de la razón instrumental moderna donde nadie es soberano. La esposa-madre representa la esencia femenina del mundo de la necesidad en el que el varón se halla atrapado. Sólo la prostituta encarna la «posición de objeto» necesaria para la experiencia de la soberanía. Como figura en la que canalizar el deseo de destrucción, la voluntad aniquiladora, su papel es muy importante. Por ello, Bataille desestima los intentos abolicionistas de la prostitución de ciertos espíritus a los que califica irónicamente de «solidarios». La prostitución, femenina y masculina en Sade, únicamente femenina en Bataille, cumple la función de receptora del odio ontológico expresado en el erotismo, evitando la generalización de la violencia y las perturbaciones del orden político. Mientras que nuestro pensador rechaza la esclavitud por considerar que el intento de reducir a los hombres a meros objetos se halla irremisiblemente condenado al fracaso por ser una impostura, una «ficción» generada por los intereses de los opresores, considera que, dada la naturaleza agresiva y dominante de los hombres, la preservación del espacio democrático de los iguales caracterizado por el universal respeto de los derechos individuales exige el sacrificio de una parte del colectivo femenino. Las mujeres, afirma, han tenido que convertirse en objetos para que exista el erotismo, constructo distinto de la espontánea sexualidad animal.

Como podemos observar, la reificación de los géneros y la propuesta sociopolítica de Bataille muestran un carácter claramente sexista. Ahora bien, continuando el análisis, intentaré detectar ese otro sesgo de género llamado androcentrismo. Si el sexismo inferioriza y tiende a discriminar o excluir a las mujeres, el androcentrismo fija estándares de lo propiamente humano a partir de la identidad masculina tal como es definida históricamente en los límites del sistema de género. Y en este punto, me parece oportuno recordar, con Seyla Benhabib, que «el sistema génerosexo es la red mediante la cual el self desarrolla una identidad incardinada, determinada forma de estar en el propio cuerpo y de vivir el cuerpo. El self deviene yo al tomar de la comunidad humana un modo de experimentar la identidad corporal psíquica, social y simbólicamente» ${ }^{8}$.

Consideraré caracteres androcéntricos de la moral de la transgresión: 1) el dualismo jerarquizado y fuertemente generizado Naturaleza/Cultura; 2) la absolutización de la Voluntad de Dominio o identificación de Eros y Thanatos; 3) el pathos trágico del héroe solitario.

\section{El dualismo generizado Naturaleza/Cultura}

El primero de los caracteres que me propongo señalar fue tempranamente explorado por Simone de Beauvoir en El Segundo Sexo. Sin abandonar la oposición y jerarquización del par Naturaleza/Cultura, observó que la cultura patriarcal condenaba a las mujeres a la inmanencia cíclica del orden natural, reservando para el varón la historicidad y el acceso al ser como proyecto propio de lo auténticamente humano. En esta misma línea, Sherry Ortner planteó la hipótesis de la universal concepción de la mujer como mediadora entre hombres y 
Naturaleza como explicación del origen del orden patriarcal. Los procesos de procreación y crianza facilitarían que se identificara a la mujer con algo generalmente devaluado: la Naturaleza. Así, afirma Bataille, «nos molesta salir de la vida, de la carne, de una inmundicia sanguinolenta [...] la materia misma, en el mismo nivel en que nos separamos de ella, es el objeto privilegiado de nuestra náusea» ${ }^{9}$. Este asco no es natural, sino propio del desarrollo de la conciencia humana y la civilización. Es rechazo de la Naturaleza en tanto ésta muestra la vida anónima e infinita de un devenir en el que vida y muerte se hallan unidas ${ }^{10}$. Como el vitalismo nietzscheano, la teoría de Bataille se opone a la tranquila y mesurada aceptación de los placeres naturales del epicureísmo.

La Naturaleza de Bataille, como la de Sade, es una madrastra que devora a sus hijos. Nos hallamos, pues, muy lejos de las bucólicas descripciones de Lucrecio en $D e$ rerum natura. Si la civilización y, particularmente, el cristianismo han procedido a la negación del mundo y de la naturaleza propia del hombre a través de la construcción de un reino de pureza regido por estrictas reglas morales, el transgresor es el hombre como Naturaleza transfigurada o segunda Naturaleza, producto de la Cultura.

Lo femenino aparece vinculado, por un lado, a la Naturaleza originaria, a las Ménades, territorio inconsciente en el que la vida y la muerte se confunden, y, por otro, a la Naturaleza maldita (Naturaleza marcada por la condena de la civilización paradigmáticamente representada por la figura de la sexualidad prohibida o prostitución). Lo masculino se presenta, en cambio, en primer lugar, relacionado con la Ley que establece un orden supranatural. Los hombres crean las reglas morales que exigen el respeto de la madre, la hermana y la esposa. Estas reglas proceden a la instauración de un mundo de pureza que los libera y aleja del horror caótico y carente de sentido del devenir. La segunda figura de lo masculino será la de la transgresión o negación de la negación de la Naturaleza.

La transgresión (en principio accesible a hombres y mujeres) será deseo de la Naturaleza maldita. No es mero retorno al origen, sino trascendente afirmación de la animalidad sagrada. En Acéphale, Bataille sugiere la relación entre su teoría materialista y el triángulo edípico: el orden establecido por Dios, los reyes y los gobiernos son obstáculos entre el hombre y la Tierra como el padre es la barrera que impide la violación de la madre por el hijo. El vínculo de amor-odio hacia la madre concebida como Naturaleza repulsiva es también un deseo de identificación con el Padre. Sólo habrá placer en la transgresión, en la sublevación contra el Padre y en la humillación de la Madre.

Pero tras la nietzscheana muerte de Dios, el sadomasoquismo se convierte en experiencia mística extática: si Dios es el Padre ausente, si es la Nada, el hijo también quiere serlo. Alcanza la totalidad a través del erotismo o sexualidad prohibida por el Padre que le permite alcanzar, al menos por algunos instantes, la vivencia de la libertad absoluta o carencia de determinaciones, es decir, la Nada. El sentimiento motor será el odio (o deseo de eliminación de las barreras del ser determinado). La aniquilación, como manifestación del dominio, debe llegar a la autoinmolación. El acceso a lo sagrado que procura el sacrificio ritual o el erotismo (entendido también como sacrificio) revela la continuidad de los seres aparentemente discontinuos. Particularmente significativa resulta la negación de la belleza de las formas naturales como deseo de mostrar su cara oculta repugnante. Las vísceras son la verdad del objeto del deseo. Resulta interesante subrayar la vigencia de esta concepción en las actuales producciones de ficción de la cultura de masas: náusea y seducción ante excrementos, sangre y entrañas, individuos sin rumbo que intentan afirmarse a través de la violencia irracional. 


\section{Absolutización de la Voluntad de Dominio}

Estrechamente ligada al rechazo de una Naturaleza feminizada y de una feminidad naturalizada se halla la exaltación de la voluntad de dominio y la elisión de los caracteres maternales presentes en la $\mathrm{Na}$ turaleza. Su materialismo, como el de Hobbes, concibe el estado de naturaleza únicamente como lucha de todos contra todos carente de cualquier tipo de simpatía, colaboración o solidaridad. El acceso a la soberanía por parte del individuo actual sólo es posible, entonces, mediante la destrucción de la «herencia de diecisiete siglos de cobardía» ${ }^{11}$. Compasión, amor, gratitud y ayuda mutua son principios serviles y artificiales que, como Sade ya mostrara, han de ser abandonados.

Es importante tener en cuenta, por su patente actualidad, que el vitalismo de Bataille implica una redefinición del modelo de virilidad: se trata de un paso del autocontrol del varón adulto regido por principios racionales al descontrol pulsional juvenil y violento de la transgresión de las reglas. Recordemos que la templanza (sophrosyne) es una cualidad requerida a los jefes en el pensamiento griego antiguo. Actitud viril de dominio de sí mismo, la templanza es virtud propia del varón libre en tanto control racional de las pasiones. Sólo quien es capaz de dominar su propia naturaleza podrá ejercer adecuadamente el dominio sobre mujeres y esclavos. Este modelo de autocontrol ejercerá gran influencia en la historia del pensamiento posterior.

Puesto que, de acuerdo al status de género, se define como viril lo que se considera superior y se considera superior lo que los varones adoptan históricamente como práctica y modelo, mientras que en Kant, siguiendo una larga tradición, la razón práctica es viril y las mujeres no logran más que un simulacro de conducta ética ${ }^{12}$, en Bataille es viril la transgresión de la norma moral efectuada gracias a la violencia de la energía pulsional propia del organismo joven. Será viril la lucha de las pasiones contra la ética del justo medio, contra lo sano, lo limpio y lo racional. Y como lo viril es considerado lo superior y propiamente humano, es también la regla y modelo neutro propuesto para todos los seres humanos. De ahí que algunas figuras femeninas paradigmáticas de Bataille, como en Sade, encarnen el erotismo sádico. Esta aparente igualdad libertina entre los sexos ha llevado a algunas teóricas a referirse a un supuesto feminismo sadeano ${ }^{13}$.

En Bataille, nuevamente a la manera de Sade, se trata de alcanzar la insensibilidad soberana, la apatía, no la mediocridad voluptuosa del que simplemente goza de los placeres de los sentidos. La soberanía exige la eliminación del apego. Ésta se logra no por el trabajo interior del ascetismo (por ejemplo, en la ética budista de los iluminados), sino por la destrucción del objeto. «El único modo de dar cumplimiento al deseo como deseo puro de deseo que coincide consigo mismo instituyéndose a título de norma y realiza de este modo la autonomía narcisista del sujeto - varón, naturalmente - es la abolición del objeto del deseo, a ser posible de forma metódica, es decir, mediante tortura» ${ }^{14}$. El estricto formalismo kantiano y el deseo puro sadeano compartirían, así, cierta visión androcéntrica de la autonomía característica de lo que Celia Amorós ha llamado «el sujeto iniciático», sujeto inverosímil o «desmadrado en el doble sentido de sujeto salido de madre y de sujeto sin madre, inengendrado y generador absoluto de sentido» ${ }^{15}$. La exigencia de eliminación del apego ha recibido interesantes tratamientos desde una perspectiva psicoanalítica feminista - por ejemplo, en los trabajos de Jessica Benjamin ${ }^{16}$ - que nos permiten trazar una clara línea de vinculación entre la construcción de la identidad sexuada masculina y ciertos aspectos de la tradición filosófica. 
El feminismo radical surgido en la década de los setenta denunció la unión de Eros y Thanatos como teoría y práctica patriarcales. Reducir el Eros a odio ontológico y Voluntad de poder implica una elisión de todos aquellos aspectos ligados a la necesidad de amor y reconocimiento. Por otro lado, se trata de una naturalización que oculta los componentes políticos de esta visión parcial de género.

\section{El pathos trágico}

Finalmente, como tercer y último elemento androcéntrico, haré una breve referencia a una de las consecuencias existenciales de la visión pesimista de la Naturaleza y de la autopercepción egocéntrica del sujeto iniciático masculino de la postmodernidad.

El héroe trágico del vitalismo de Bataille es un hombre desgarrado entre Naturaleza y Cultura. Su tragedia reside en no poder ser ninguno de los dos seres caracterizados por la completitud: no es ni Dios, ni animal ${ }^{17}$. La «moral inmanente» consistirá en reír ante la Nada y asumir la muerte sin apelar al bien de un ser supremo (sea éste Dios o el Estado). Dada la negación de las redes morales vinculadas al sentimiento de solidaridad y de reconocimiento de los demás, este hombre se halla solo frente al vacío, desarraigado en un mundo sin sentido. El materialismo de Bataille, por su fuerte sesgo androcéntrico, no es capaz de proporcionar consuelo frente a la pérdida del Padre y de su Ley ni frente a la conciencia de la muerte como destino humano.

\section{Volviendo a los interrogantes iniciales}

Los ejes fundamentales de la «moral inmanente» de Bataille gozan de una curiosa vigencia en numerosos fenómenos de la cultura occidental actual. En algunas oca- siones se trata de una influencia directa de este pensador pero, por lo general, la coincidencia se debe a causas más profundas: Bataille habría expresado tempranamente en el discurso filosófico algunas transformaciones actuales del sistema de género.

No es casualidad si, en un período histórico en el que las mujeres comienzan a ver reconocidas sus antiguas reivindicaciones ilustradas de los derechos, surge un nuevo discurso sobre la identidad viril que exhalta la violencia de la transgresión. La oposición entre una jurisprudencia que manifiesta cada vez menor tolerancia ante la violencia sexual y la cuasi perpetua exhibición de ésta en las producciones de la cultura del ocio puede ser entendida como política de resistencia patriarcal o simple compensación fantasmática. Quizás sea ambas cosas a la vez.

Cuando las mujeres comienzan a acceder al estatuto de sujeto, se anuncia que éste se disgrega en infinitos fragmentos dispersos e incoherentes ${ }^{18}$. En su lugar, el modelo de la hybris es presentado como paradigma de la autenticidad humana. Como la Mujer-Naturaleza maternal rousseauniana ha sido impugnada ${ }^{19}$, se la sustituye por la Mujer-Naturaleza maldita de Bataille. En la sociedad competitiva del capitalismo de consumo, la antigua figura de la seductora rejuvenece en los rasgos de la audaz transgresora ${ }^{20}$. Transgresión pero no revolución.

Esta pseudoigualdad transgresiva no es sino la adopción acrítica de un modelo androcéntrico. La moral de la transgresión expresa la rebelión nihilista de unos hijos que continúan existencialmente anclados en el dualismo generizado y jerarquizado Naturaleza/Cultura que fundara ese Padre al que declaran muerto. El afán de separación y trascendencia de esta paradójica moral inmanente desemboca en el pathos trágico. Su propuesta continúa desvalorizando actitudes y valores tradicionalmente femeninos y exaltando aquellos históricamente masculinos. La transgresión que 
ataca las normas de la herencia ilustrada es el intento de retorno a un antiguo orden patriarcal al que las nuevas condiciones socioeconómicas y culturales dan un carácter espasmódico y a-contractual.

Ante esta situación, la ética y la filosofía política feministas han de reivindicar la igualdad entre los sexos, pero proceder también a una crítica del androcentrismo. Ambas tareas, si son entendidas como proyectos excluyentes, encierran ciertos peli- gros. Así como las éticas del cuidado pueden derivar en el conformismo y en la exaltación de virtudes producidas por el sometimiento, la asunción acrítica de una transgresión pseudoliberadora implica la aceptación de valores que esconden un subtexto de género. Su análisis nos muestra que desertar las filas de una razón y una moralidad que comienza a respetarnos como individuos no es, probablemente, nuestra mejor opción.

\section{NOTAS}

\begin{abstract}
${ }^{1}$ Este trabajo se enmarca en el Proyecto de Investigación Acciones y actitudes de las mujeres frente al medio ambiente. Perspectivas teóricas y proyecciones de futuro del Programa Sectorial de Estudios de las Mujeres y del Género, Plan Nacional de I+D.

2 A. H. Puleo, Dialéctica de la sexualidad. Género y sexo en la Filosofía contemporánea, Madrid, Cátedra, 1992.
\end{abstract}

${ }^{3}$ G. Bataille, Sur Nietzsche. Oeuvres Complètes, t. VI, Gallimard, París, 1973, p. 49. La traducción es nuestra.

${ }^{4}$ Ulrich Beck, La sociedad del riesgo, trad. J. Navarro, D. Jiménez y M. R. Borrás, Barcelona, Paidós, 1998.

5 G. Bataille, Sur Nietzsche, ed. cit., p. 45.

6 Jean-Paul Sartre, Situations, I, París, Gallimard, 1947, pp. 174-229.

7 Amelia Valcárcel, Sexo y Filosofía. Sobre «Mujer» $y$ «Poder», Barcelona, Anthropos, 1991.

8 Seyla Benhabib, «El Otro generalizado y el Otro concreto», en Teoría feminista y Teoría crítica. Ensayos sobre la política de género en las sociedades de capitalismo tardio, Edicions Alfons el Magnànim, Generalitat Valenciana, 1990, p. 125.

${ }^{9}$ G. Bataille, H. E., O. C., t. VIII, p. 52. La traducción es nuestra.

${ }^{10}$ Ibid., pp. 53 y 68-70.

11 G. Bataille, H. E., O. C., ed. cit., p. 154.

12 Concha Roldán, «El reino de los fines y su gineceo: las limitaciones del universalismo kantiano a la luz de sus concepciones antropológicas», en Roberto, Aramayo, Javier Muguerza y Antonio Valdecantos (comps.), El individuo y la historia. Antinomias de la herencia moderna, Barcelona, Paidós, 1995.

${ }_{13}$ Para la refutación del supuesto feminismo de Sade, ver Luisa Posada Kubissa, Sexo y esencia. Sobre esencialismos encubiertos y esencialismos heredados. Por un feminismo nominalista, Madrid, Horas y Horas, 1998, pp. 37-64.

${ }_{14}$ Celia Amorós, Tiempo de feminismo. Sobre feminismo, proyecto ilustrado y postmodernidad, Madrid, Cátedra, 1997, p. 220.

15 C. Amorós, op. cit., p. 21.

16 Ver particularmente J. Benjamin, Los lazos del amor. Psicoanálisis, feminismo y el problema de la dominación, trad. Jorge Piatigorsky, Barcelona, Paidós, 1996.

${ }^{17}$ G. Bataille, O. C., t. V, p. 269.

18 Sobre el feminismo como refutación de la muerte del sujeto, entendiendo por sujeto aquel que «pueda interrumpir reflexivamente la cadena del significado construida tomando distancia crítica y alterándola creativamente», ver Cèlia Amorós, «Feminismo, Ilustración y Postmodernidad. Notas para un debate», en C. Amorós (coord.), Historia de la Teoría feminista, Instituto de Investigaciones Feministas, Universidad Complutense de Madrid, 1994, p. 348.

19 Ana de Miguel ha visto en las primeras críticas feministas al modelo de la virtuosa mujer doméstica una «Sociología olvidada» que descubrió la naturalización de la mujer como mecanismo legitimador de la desigualdad sexual (Ana de Miguel, «La Sociología olvidada: género y socialización en el desarrollo de la perspectiva sociológica», en Política y Sociedad, núm. 32, 1999, septiembre-diciembre, número monográfico Género y Ciencias Sociales, Facultad de Ciencias Políticas y Sociología, Universidad Complutense de Madrid, pp. 161-171.

${ }^{20}$ Agradezco a la profesora de Historia del Arte María Teresa Alario, de la Cátedra de Estudios de Género de la Universidad de Valladolid, sus observaciones sobre la hegemonía y características de esta figura de la seductora en el discurso actual de la publicidad. 